\title{
LE CINQUE GIORNATE DI ALFONSO LITTA MODIGNANI
}

\author{
Nota del s.c. GIANMARCO GASPARI (*)
}

(Adunanza del 5 maggio 2016)

SuNTO. - La recente pubblicazione, curata da Claudia Locatelli e coordinata da Antoniotto Guidobono Cavalchini, del diario manoscritto sul proprio coinvolgimento nella prima fase delle Cinque giornate di Milano, che Alfonso Litta Modignani lasciò in custodia della famiglia, rappresenta una notevole testimonianza storica degli eventi e restituisce insieme al lettore una dimensione di singolare partecipazione umana. Da rilevare, in una lettura che procede per rapide campionature, la qualità letteraria di questa preziosa trouvaille, che più di un referto ci fa scoprire particolarmente vicina al magistero manzoniano.

$* * *$

ABSTRACT. - Alfonso Litta Modignani had a major role during the Five Days of Milan. He gave an account of this intense though brief experience in a diary, later kept in his family archive, and now edited by Claudia Locatelli and Antoniotto Guidobono Cavalchini. The diary is a remarkable historical record of the 1848 events and it offers to the reader a unique dimension of human involvement. It is also worth mentioning the literary quality of this precious discovery, particularly close - as several clues reveal - to the lesson of Manzoni.

(*) Istituto Lombardo-Accademia di Scienze e Lettere, Milano; Università degli Studi dell'Insubria, Varese, Italia. E-mail: gianmarco.gaspari@uninsubria.it 
Il 17 marzo 1848 era - guarda caso - un venerdì. Don Alfonso Litta Modignani aveva allora trentasette anni ed era felicemente sposato da pochi mesi. Quel venerdì ascoltò a lungo, dalla sua casa di contrada della Passarella (sull'area che dalla contrada del Durino andava a incrociare a nord il corso di Porta Orientale; la casa sopravvive tutt'oggi, in corso Europa) il brusio insistente che percorreva le strade del centro. Si era sparsa la voce di un'insurrezione a Vienna: e l'informazione parve confermarsi la mattina dopo, quando sulle cantonate si lessero degli avvisi ufficiali che dichiaravano ripristinata la libertà di stampa. Alfonso aveva esperienza di governo, essendo stato nel 1840-42 tra i consiglieri del Comune. Poteva, lui come numerosi altri, legittimamente credere che un atto del genere venisse a segnare la fine della dominazione asburgica, o almeno avviarla. Del resto, forse era già arrivata voce a Milano della sollevazione ungherese, scoppiata due giorni prima. Ma la «primavera dei popoli» dilagava a macchia d'olio, dalla Prussia a Praga. Già a febbraio, la rivolta di Parigi era riuscita a ottenere l'abdicazione di Luigi Filippo. Era il turno di Milano.

Il 18 marzo segna l'avvio delle Cinque giornate. Ritrovandola tra le carte di famiglia, Antoniotto Guidobono Cavalchini ha pubblicato ora la testimonianza di Alfonso Litta Modignani, che visse di persona le ansie della vigilia, si offerse alla causa, rischiò la vita, venne incarcerato e fortunosamente liberato. ${ }^{1}$ Si tratta di una breve memoria, limitata a queste sole circostanze e chiusa dunque dalla scarcerazione, il 19 marzo. La prospettiva limitata scelta dall'autore - tutta centrata sulla propria esperienza e funzionalmente assimilabile alla tipologia del diario -, insieme con l'asciuttezza della prosa, colta ma efficace, e soprattutto priva di ogni tentazione retorica (cui, come ben sanno gli storici, la memorialistica risorgimentale è particolarmente esposta), impongono questo scritto come uno dei più notevoli entro la numerosa produzione coeva. La pubblicazione, ci informa la breve Prefazione, è condotta sulla base del testo trascritto dalla figlia minore di Alfonso, Annetta (per offrire in lettura queste «note dei memorabili giorni» del marzo 1848 ai suoi «carissimi figlioli a ricordo del loro ottimo nonno»); «un'altra versione della cronaca», di mano della figlia maggiore, Clementina, si trova ora in una residenza privata di famiglia, a Morazzone: se tra le carte domestiche sia conservato anche il testo originale, quali siano le differenze tra le due trascrizioni e quali interventi

1 Le cinque giornate di Alfonso Litta Modignani, a cura di C. Locatelli. Acquerelli di N. Astakhova, Milano, Proedi, 2015. 
siano stati effettuati sul testo (che sia avvia tronco, con una frase preceduta da puntini, e in più luoghi presta il fianco a qualche dubbio sulla lettura corretta), non è dato sapere, ma ci troviamo - lo si sarà inteso - di fronte a un'operazione che è stata condotta in forma di omaggio privato, e fatta circolare al di fuori del circuito commerciale. Un dettagliato Albero genealogico della famiglia Litta Modignani, curato da Alessandro Parrocchetti Piantanida, chiude il volume.

Per meglio avvicinare la personalità dell'autore (che in quanto tale ci è noto solo tramite questo scritto), può risultare interessante, proprio all'avvio del testo, la considerazione che Alfonso lascia cadere a proposito del fermento presente in città dalla vigilia, appunto da quel venerdì: a séguito degli «avvenimenti di Francia», scrive, l'agitazione cresceva, e «nei moltissimi raddoppiava le speranze, nei pochi il timore, in tutti un'ansia, una certezza che qualcosa d'incognito ma di nuovo e di straordinario sarebbe avvenuto» (p. 13, mio il corsivo). Non credo si vada troppo lontano dal vero mettendo in conto al memorialista il ricordo di un luogo celebre del romanzo manzoniano: mi riferisco al cap. XII, che muovendo dalla cronaca del 1628, il «second'anno di raccolta scarsa», in una situazione di tensione che vedeva la capitale privata di un'autorità all'altezza dell'emergenza (il governatore e gran parte della forza pubblica della città erano impegnati nello sfiancante assedio di Casale), disegna il terreno fertile che andava guadagnando il progetto di rivolta. Si ponga mente soprattutto al primo periodo che qui stralciamo, con l'insistenza sul tema della moltitudine ( $\mathrm{i}$ «moltissimi» del diario di Alfonso) fino ai dettagli della resa descrittiva, connotata da frasi frante e, alla fine dello stesso periodo, dalla scelta (peraltro non così comune) della forma narrativa del condizionale (che qui pure evidenzio con il corsivo):

Migliaia d'uomini andarono a letto col sentimento indeterminato che qualche cosa bisognava fare, che qualche cosa si farebbe. Avanti giorno, le strade eran di nuovo sparse di crocchi: fanciulli, donne, uomini, vecchi, operai, poveri, si radunavano a sorte: qui era un bisbiglio confuso di molte voci; là uno predicava, e gli altri applaudivano; questo faceva al più vicino la stessa domanda ch'era allora stata fatta a lui; quest'altro ripeteva l'esclamazione che s'era sentita risonare agli orecchi; per tutto lamenti, minacce, maraviglie: un piccol numero di vocaboli era il materiale di tanti discorsi.

La descrizione manzoniana cavalca il discrimine sottile che separa l'evidenza dalla suggestione, per farsi veicolo di un'ipotesi di analisi 
sociale che l'Ottocento avrebbe codificato come "psicologia della folla", ma che poteva fino ad allora esibire ben pochi precedenti. Che la prosa di Manzoni (si è citato dall'edizione definitiva del romanzo, quella del $1840-42)^{2}$ sia particolarmente attenta alle dinamiche sottese a questo tema, è dato che difficilmente sfugge al lettore del romanzo, che penetrerà bene questi recessi nelle documentatissime pagine storiche (a quelle già richiamate, come è noto, è da aggiungere l'intera sequenza dei capp. XXXI-XXXIV). ${ }^{3}$ E nella Colonna infame, dove il peso della storia schiaccia a terra ogni lusinga di invenzione, la larga parte occupata dall'analisi delle suggestioni collettive non si esimerà dal rievocare, nel cap. IV, persino le «turbe» che consegnarono Cristo alla morte, con calco diretto dal testo latino: «Exclamavit autem simul universa turba, dicens: Tolle!» (Luc. XXIII 18).

Con la sua Histoire de la Révolution française, sarà Jules Michelet a introdurre la prospettiva moderna che imporrà risolutamente questo come tema centrale nella storiografia dell'Otto e poi del Novecento. Merita infatti ricordare come la moderna storia d'Europa incontri appunto la folla solo dopo la grande Rivoluzione di Francia: e si tratta ovviamente della folla urbana, non certo degli assembramenti che nelle campagne riunivano periodicamente le moltitudini nelle processioni o nelle fiere. È la folla urbana quella che si aggrega obbedendo a un richiamo, tacito o esplicito, in dipendenza dal paradigma che si definisce a Parigi nel luglio dell'89. Ma il romanzo manzoniano non si nega, anzi, precede di non poco questa riflessione, sino alla conseguente mutuazione, nel dettaglio, di un termine come massa (presente quattro volte nell'edizione definitiva, salve le locuzioni del tipo in massa) dal linguaggio della storiografia d'inizio secolo, a designare precisamente quel nuovo soggetto politico. Anche Rosmini (lo ha ricordato qualche tempo fa Mario D'Addio) ${ }^{4}$ tentò di sviluppare una teoria della «ragion

2 A. Manzoni, I Promessi sposi, vol. II (I Promessi sposi nelle due edizioni del 1840 e del 1825-27 raffrontate tra loro. Storia della Colonna infame) a cura di L. Caretti, Torino, Einaudi, 1971, 282-283. Le varianti tra le due edizioni sono per questa parte irrilevanti rispetto alla nostra analisi.

3 Cenni a questo "trattamento" del tema, in relazione all'ultimo cap. richiamato, nel mio contributo Nella città dolente, in P. Fandella, G. Langella, P. Frare (a cura di), «Questo matrimonio non s'ba da fare». Lettura de «I Promessi sposi», Milano, Vita e Pensiero, 2005, 143-148.

4 M. D’Addio, Manzoni politico, Lungro di Cosenza, Marco, 2005, passim. 
pratica delle masse», ai fini di una miglior comprensione delle dinamiche sociali, nel terzo libro della Filosofia della politica. Ma Manzoni è già sulla via della riflessione sui meccanismi impliciti dell'adesione che percorreranno poi, appunto Michelet («L'attacco alla Bastiglia non fu per nulla ragionevole. Fu un atto di fede. Nessuno lo propose. Ma tutti credettero, e tutti agirono. Lungo le vie, sui lungofiume, dai ponti, dai boulevard, la folla gridava alla folla: "Alla Bastiglia! Alla Bastiglia ..." [...] Nessuno, ripeto, diede l'impulso») $)^{5}$ e, nel Novecento, in prospettiva complementare e con non minore tragicità, il Canetti di Massa e potere e de Il frutto del fuoco (qui, a proposito della rivolta di Vienna del 15 luglio 1927: «La reazione fu assolutamente spontanea, me ne accorsi più che mai dai miei sentimenti. Inforcai la bicicletta, volai in città e mi unii a uno di quei cortei. Gli operai, che normalmente erano disciplinati, avevano fiducia nei loro capi del partito socialdemocratico e si dichiaravano soddisfatti del modo esemplare in cui essi amministravano il Comune di Vienna, agirono in quel giorno senza consultare i loro capi.»), ${ }^{6}$ la stessa via che lo condurrà più avanti ad approfondire, anche empiricamente e sempre a margine del suo mai sopito interesse per la rivoluzione di Francia, l'influenza della «tirannia dell'opinione» (tema che avvicinerà poi un altro diretto protagonista delle Cinque giornate, Carlo Cattaneo, nello studio, rimasto incompiuto, sulla Psicologia delle menti associate). Riflessioni che avrebbero portato lontano (e se Michelet dedicò un intero volume della sua monumentale Histoire de la Révolution a Le peuple, ci mantiene più prossimi a Manzoni la presenza della folla urbana, nell'immaginaria ma "parlante" Cocktown, folla questa volta di operai disillusi e pronti alla violenza fino ad allora inaudita dello sciopero, in un romanzo di Dickens del 1844, Tempi difficili, che più di uno spunto dovette suggerire a Fritz Lang per la sceneggiatura di Metropolis). ${ }^{?}$

Con questo, resta chiaro che anche solo l'avvicinamento di un dilettante della scrittura come Alfonso Litta Modignani a questa messa a fuoco identifica una recettività assolutamente fuori del comune. E che non si tratti di mera casualità, lo potremmo verificare muovendo verso

5 J. Michelet, Storia della Rivoluzione Francese, Milano, Rizzoli, 1981, 107.

6 E. Canetti, Il frutto del fuoco, Milano, Adelphi, 1982, 249.

7 Per questi riferimenti si veda G. Gaspari, Manzoni, la storia e l'Europa romantica, Nuova Antologia 153 (2018), 243-258. 
la fine della sua narrazione, quando l'inattesa (e rapida) liberazione lo vede transitare dalla contrada di San Nicolao per approdare, sboccato sul Corso di Porta Vercellina, al desiderato rifugio di Palazzo Litta: «...mi parve di entrare in una città abbandonata: chiuse tutte le imposte e le gelosie, non il minimo lume che trapelasse dalle finestre, non un segno di vita tranne il rumore delle mie pedate rimbombanti in quel solenne silenzio» (p. 55). Un brano dove la ripresa dello stesso tema, in diversa chiave, non può non associare la struttura ritmica del periodo e il suo movimento per ellissi alla pagina celebre nella quale Manzoni rievocava la desolazione della città all'indomani del tumulto di San Martino, nel cap. XXVIII:

A ogni passo, botteghe chiuse; le fabbriche in gran parte deserte; le strade, un indicibile spettacolo, un corso incessante di miserie, un soggiorno perpetuo di patimenti...

Basterebbero questi dettagli a indurci a una verifica, per la verità comunque opportuna, sui rapporti diretti tra Alfonso e Manzoni. Che sono certificati anche da parentela, dato che il terzo dei nove fratelli di Alfonso, Lorenzo (nato nel 1797, dunque maggiore di quattordici anni di Alfonso, e di dodici anni minore di Manzoni) aveva sposato nel 1826 Carolina Trotti Bentivoglio: Carolina era sorella di Lodovico, che nel 1838 aveva a sua volta sposato Sofia Manzoni, la quinta figlia dello scrittore. L'epistolario di Manzoni documenta con puntualità la consuetudine tra le due famiglie. Sappiamo così che nell'autunno del 1829 il primogenito maschio di Manzoni, Pietro, era ospite a Biumo, sopra Varese, nella villa di Giovan Battista Litta: e che proprio Lorenzo, a quella data, si era già interessato alla nascente filosofia rosminiana, tanto che Manzoni si dice ansioso di parlare con lui del Nuovo saggio sull'origine delle idee, ancora fresco di stampa, che Rosmini stesso aveva

8 Manzoni, I Promessi sposi, vol. II, 640 (vale quanto detto sopra per le differenze tra prima e seconda edizione). Si noti che nel diario di Alfonso la locuzione temporale con cui si avvia il passo manzoniano era pure presente, poco sopra, proprio a inizio periodo e in un contesto analogo: «A ogni passo dunque, vedendomi andare in fretta e con una carta in mano, mi fermavano per dmandarmi se c'erano notizie e a che punto stavano le cose...» (p. 19).

9 A. Manzoni, Tutte le lettere, a cura di C. Arieti, con un'aggiunta di lettere inedite o disperse a cura di D. Isella, Milano, Adelphi, 1986, t. I, 570-571. 
provveduto a inviare ad entrambi. ${ }^{9}$ Anche il quarto dei fratelli Litta, Luigi (nato nel 1803), è presente nelle lettere manzoniane, per la parte avuta nel tentativo di impedire a Napoli la diffusione, nel 1841, di copie-pirata dell'edizione illustrata del romanzo, dati i buoni rapporti intrattenuti con la diplomazia austriaca. ${ }^{10}$

Più curiosa la menzione del quintogenito, Alessandro, che ha consegnato alla storia la fisionomia di un appassionato e attento viaggiatore: tra il 1838 e il 1852 visitò l'Armenia, la Siria, l'Arabia (toccando anche la città di Petra), la Terra Santa, l'Egitto e, nel 1841, l'America meridionale: il Brasile, l'Argentina, il Cile, la Bolivia, il Perù e la Giamaica. Al ritorno da quest'ultimo viaggio Alessandro portò in dono a Manzoni, con il quale condivideva l'entusiastica passione per la botanica, dei semi di ceroxylon, un palmizio originario delle Ande. Manzoni conosceva bene la pianta, che aveva incontrato nella monografia di Alexander von Humboldt sui Nova genera plantarum: così, quando nel giugno del 1844 lo stesso Humboldt, nella sua qualità di cancelliere dell'Ordine per il merito nelle Scienze e nelle Arti del Regno di Prussia, gli scrisse per annunciargli la nomina a cavaliere, lo scrittore rifiutò, come era solito fare per non dare adito alla possibilità che l'Austria gli offrisse a sua volta qualche onorificenza - che non sarebbe riuscita gradita -, ma approfittò della ghiotta occasione per chiedere al grande scienziato tedesco qualche informazione sulla crescita del ceroxylon che ricorda appunto giunto a lui dalle mani di «Mon concitoyen, le comte Litta Modignani», a séguito del viaggio compiuto «guidé surtout par vous, dans l'Amérique méridionale» - , del quale con lentezza, troppa lentezza rispetto all'attesa, vedeva spuntare «un léger renflement» sulla superificie del terreno. ${ }^{11}$

Non ci è noto l'esito della vicenda, che sicuramente prova come la maggior considerazione tributata a un uomo di scienza, per Manzoni, consistesse nel coinvolgerlo fattivamente nei propri interessi, e tanto meglio quanto più pragmatici. Se un caso analogo può venire alla mente - utile forse, più che a dar la misura della rusticitas surreale sottesa ai

10 Manzoni, Tutte le lettere, t. II, 171, 188-190.

11 Manzoni, Tutte le lettere, t. II, 336-337. Per i dati, M. e L. Corgnati, Alessandro Manzoni «fattore di Brusuglio», prefazione di G. Vigorelli, Milano, Mursia, 1984, 87-88, e (anche per il successivo cenno a Morselli) G. Gaspari, Manzoni botanico e nomenclatore, Studi sul Settecento e l'Ottocento 13 (2018), 71-88. 
devoti di passione così assoluta e totalizzante, a sottolineare appunto il singolare pragmatismo dell'uomo di lettere lombardo - è quello che s'incontra tra le «lettere ritrovate» del varesino Guido Morselli, rese recentemente note, una delle quali, del settembre 1968, è indirizzata nientemeno che a Konrad Lorenz. La traduzione italiana de L'anello di re Salomone era stata pubblicata l'anno prima, e la lettera (puntualmente conservata nella copia del libro posseduta da Morselli) è scritta dal podere di Santa Trinita. In francese, l'autore di Roma senza papa chiede lumi a Lorenz sulle misure da prendere contro $i$ ghiri che gli infestavano la casa. La risposta, che Morselli ricevette nel giro di poche settimane, su carta intestata del Max-Plank Institut e firmata da un assistente dell'etologo austriaco, lapidariamente riferiva di come al momento non fosse possibile al futuro Premio Nobel offrire «alcun consiglio su come si possano cacciare i ghiri».

Un mondo distante toto coelo dalla plumbea e terrorizzante realtà che portò Alfonso all'imprigionamento al Castello, la sera del 17 marzo 1848. E che segna un ulteriore percorso parallelo alla biografia di Manzoni: il suo penultimo figlio, Filippo, sabato 18 (compiva allora i ventidue anni), venne arrestato al Broletto, dove si era recato per arruolarsi nella Guardia civica, e trattenuto quindi al Castello fino alla notte del 23, quando iniziò il viaggio che da Melegnano a Verona e Rovereto lo condusse al campo di prigionia di Kufstein, in Tirolo, dove, seguito dall'ansia costante della famiglia, rimase fino alla fine di maggio. In agosto, dopo aver salutato il padre a Lesa, riparò a Lugano, per unirsi ai molti altri esuli - da Giuseppe Mazzini a Giovanni Visconti Venosta che avevano vissuto quei giorni alterni di speranza e delusione. ${ }^{12}$

Al Broletto Alfonso Litta Modignani era dunque giunto prima del giovane Manzoni, la sera di quel venerdì 17, in tempo per partecipare alla riunione preparatoria che aveva il compito di organizzare l'azione antiaustriaca. Si trattava di irreggimentare una «guardia urbana», in un clima che si andava facendo di ora in ora più euforico. Il nostro narratore si muove verso la mèta accompagnato dai cori di popolo, «Viva l'Italia! Viva Pio IX!», e avverte presente nei milanesi «un pensiero, una preoccupazione comune», che esprime in una parola, fratellanza, anche se (glossa con finezza) l'esistenza della «cosa» era evidente senza «che s'u-

12 La vicenda di Filippo viene riscostruita in A. Stella e G. Gaspari (a cura di), Voci e volti di Casa Manzoni, Milano, Centro Nazionale Studi Manzoniani, 75-84. 
disse il nome» (p. 19): pronunciare quella parola, troppo legata all'eco durevole della grande Rivoluzione di Francia, era di fatto assai pericoloso. E del resto, quando dallo sbocco di via San Martino giunge alla corsia dei Servi (l'attuale corso Vittorio Emanuele), ecco i primi colpi di fucile, come gli chiarirà subito dopo un anonimo interlocutore: «Badi che ci sono i Tirolesi sul Duomo che sparano addosso a quelli che si fanno vedere sulla Corsia» (p. 23). Per arduo che fosse, dal coperto dei Figini svolta verso l'attuale Cordusio e approda così al Broletto, dove nella Sala del Podestà erano già riuniti gli organizzatori della rivolta. Nel momento in cui Alfonso entra nella sala, «senza che ci fosse il bisogno di chiedere il silenzio», il delegato provinciale Bellati attira su di sé l'attenzione di tutti annunciando di avere appena ricevuto un messaggio del generale Radetzky, e ne dà lettura. Come è noto, gli austriaci chiedevano la resa senza condizioni, minacciando l'arrivo in città, per il giorno dopo, di una guanigione di duecentomila uomini «a castigare la sediziosa città» (p. 24).

La discussione che subito ne seguì - polarizzata sulla scelta di avviare subito la rivolta, certi che quello di Radetzky fosse un bluff, e la tentazione di «cedere dinanzi al pericolo» - viene interrotta da un formidabile movimento proveniente dall'esterno. Notevole, da questo punto in poi, da quando cioè la narrazione inizia a far perno sulla sensibilità e sul totale coinvolgimento del protagonista, la resa descrittiva messa in carta da Alfonso, una sorta di "presa diretta" di raro talento. $\mathrm{Ne}$ citiamo un passo saliente, nel quale è l'approssimarsi del rumore, prima appena avvertito e poi impressionante nella sua vicinanza, ad apparentare queste pagine di diario a una sorta di affondo da gothic novel, perfettamente padroneggiato nei suoi artifici (pp. 24-25):

Già da qualche tempo anche in mezzo al frastuono e al tramestio di tanta gente nella sala, nel Palazzo e fuori, m'era parso di distinguere di tratto in tratto un rumore più lontano, che non era né voci della moltitudine, né l'andare e il venire di questa, né quel'indeterminato ronzio e sussurro che sorge dove si trova adunata una grande quantità di gente, ma sebbene qualcosa di più secco come farebbe la gragnola quando batte con violenza sui tetti. Ma chi badava alla diversità dei rumori del di fuori! Ora poi, mentre stavasi discutendo in che conto dovevansi tenere le minacce di Radetzky, questo rumore si avvicinò in modo tale da non lasciare più dubbio su quel che fosse, e tutti si accorsero purtroppo che queste erano belle e buone scariche d'archibugi, fatte dalla truppa che s'avanzava verso il Broletto, e non passò gran tempo che il rumore prima avvertito dominò tutti gli altri, anche perché di mano in mano che questo progrediva, l'altro, voglio dire quello delle moltitudi- 
ni, andava dissipandosi. Questa certezza troncò tutte le discussioni e mise un gran movimento nella nostra sala e nelle adiacenti. Molti presero il cappello e se la svignarono. Altri, più arditi, proposero di difendersi dalle finestre con sassi e con quanto capitava alle mani, e di salire sui tetti facendo piovere tegoli a furia.

La stessa tecnica, una gradatio enfatica di notevole efficacia, la ritroviamo a giro di pagina, quando le truppe austriache premono ormai alla porta e si fanno innanzi, scesa ormai la notte, ai rivoltosi. In questo caso non sfuggirà al lettore l'attenzione sui dettagli luministici, quasi alla Goya, che ci fanno intuire come ai tentennamenti di quelle precarie iridescenze corrispondesse l'ansia altalenante - ma ormai rassegnata alla precoce sconfitta - dei milanesi (pp. 28-29):

Il fracasso al di fuori andava sempre crescendo e già due altri fortissimi scoppi pari al primo ci indicavano che si voleva atterrare col cannone o una porta o un pezzo di muro... poi s'ode al basso come un rovinio di di roba atterrata e sconquassata... quindi ci arrivano all'orecchio delle voci rapide e di comando: finalmente su per le scale uno strepito d'armi e di pedate frettolose e pesanti in un, con grida e colpi di fucile. In quel punto, non so se a caso o apposta, fu spento l'unico lume che rischiarava la nostra stanza, e nel perfetto buio, sospesa quasi la respirazione, nessuno entrando avrebbe potuto immaginarsi che c'erano qui tante persone. In men che il dico, il tumulto, gli urli della soldatesca, le voci imperiose degli ufficiali, lo scoppio dei fucili empirono tutto il palazzo e il sottoposto cortile... da noi si spalanca l'uscio dirimpetto tutt'a un tratto e contemporaneamente l'altro che ci stava di fianco. Da questo entra, seguito da altri, un soldato con un lume acceso, e dall'altro si precipitan con baionetta bassa altri soldati, verso di noi urlando...

L'insistenza sulle qualità letterarie del testo non metterà in ombra, in quelle che restano in primo luogo le pagine di un diario privato, la fortissima tensione di chi sa bene che la possibilità di scrivere gli è stata offerta dalla sorte, al contrario dei tanti suoi compagni cui l'esito di quella vicenda l'aveva negata; la scrittura è per Alfonso risarcimento e insieme testimonianza, e s'intende che il fatto di perseguirne (e arrivare a raggiungerlo) un livello particolarmente alto, si identifica per il diarista con un preciso compito etico. Da qui l'umanità di queste pagine: che si aprono a minuti dettagli, di un gusto quasi pittorico - sulla scia della nascente sensiblerie romantica -, come nel caso del passaggio 
rapido degli arrestati nelle sale del palazzo, quando vengono riuniti per la deportazione nell'appartamento del delegato provinciale, e lo sguardo di Alfonso cade sulla bimba che, in un angolo, dorme inconsapevole di quanto le sta accadendo intorno (p. 31):

Attarversate varie sale occupate dai soldati, ci condussero nell'appartamento del delegato Bellati, ove si trovavano raccolti tutti gli altri nostri. Qui c'erano vari ufficiali superiori che frugavano dappertutto e notavano tutto. In una stanza affollata, oppresso dall'afa e dall'emozione, mi ritirai nel vano di una finestra, accanto a un letticciolo in cui stava una bambinetta del Bellati, la quale, ad onta dello schiamazzo, dormiva come se niente fosse, con un sembiante così sereno e composto alla calma da contrastare singolarmente con le nostre facce torbide e irrequiete: la guardavo con un misto d'indivia e di tenerezza, quando un ufficiale trafelato si avvicinò alla finestra per aprire le persiane e rinfrescare l'oppressiva atmosfera, ma non le ebbe appena smosse che partirono dal basso (ove era la truppa piena di sospetti) tre o quattro fucilate contro la finestra, cosa che lo obbligò a ritirarsi in fretta bestemmiando e imprecando.

O come quando viene messa in rilievo, a dispetto della situazione, la componente umana degli aguzzini. Si tratta di un'osservazione che cade frequentemente sotto la penna di Alfonso, e che, accostata ai brani che si sono trascelti finora, ci permette di mettere a fuoco una sua precisa cifra stilistica: l'uso del contrasto come chiave descrittiva, cioè (nel passo appena citato lo si è visto esplicitato: «da contrastare singolarmente...»), che si presta perfettamente a rendere palpabili il turbamento e l'angoscia dei cittadini coinvolti nel drammatico evento. Con una breve sequenza di esempi in questo senso ci avviamo a concludere, non senza aver rilevato però, sul piano stilistico e formale, la precisa aderenza di questi moduli espressivi al sensismo di matrice razionalista. Base al tessuto narrativo di Alfonso è infatti la ricerca di effetti (tra cui appunto il contrasto) che generino sensazioni e impressioni, nel solco di una pratica estetica che in Lombardia aveva avuto particolare fortuna, tra il Beccaria delle Ricerche intorno alla natura dello stile e il romanzo manzoniano, specie in relazione alla rappresen-

13 Per tutto ciò, rinvio al mio Raccontare le passioni. Strategie narrative del sensismo nei «Promessi sposi», in G. Oliva (a cura di), Manzoni e il realismo europeo (Atti del Convegno di Chieti, novembre 2005), Milano, Bruno Mondadori, 2007, 127-142. 
tazione delle passioni. ${ }^{13}$ Esemplare a questo proposito il passo che descrive, a notte ormai inoltrata, l'arrivo al Castello, sede della prigionia dei milanesi insorti (p. 39):

Giunta la sventurata comitiva presso quella milizia, e questa vista al chiaror diffuso dei fuochi di cosa si trattava, cominciò a gridare: "Evviva! Bravi!" e a batter le mani. La fedeltà storica m'impone, obbligo penoso in questo caso, di dire che quei soldati erano italiani. Limpressione fu dolorosa.

S'entra finalmente nel cortile del castello e facciamo alto a mano destra, dove, dinanzi ad una porticina che dà l'adito su una scaletta, stava un gruppo d'ufficiali d'ogni arme; e al di sopra della ringhiera che gira intorno al fabbricato, altri ufficiali uscivano e entravano in una stanza illuminata dove credo che ci fosse il comandante in capo, cioè Radetzky. Il nostro Generale sale sopra. Al giungere nostro, quegli ufficiali si mossero tutti come spinti dalla curiosità e uno di essi, staccatosi dagli altri, s'accostò a noi e col braccio e coll'indice tesi verso la piazza del castello, mormorò tra i denti con una faccia arrovellata: "Adesso là tutti in ginocchio e..." qui, mettendo la mano destra all'occhio e l'altra semiaperta davanti a quella, come in atto di prendere la mira, espresse chiaramente quello che voleva farci intendere.

Il meccanismo narrativo che si è sintetizzato nella formula del contrasto non si attiverà nell'immediato, ma lascia ancora in sospeso il lettore che viene messo innanzi all'episodio culminante del racconto, la segregazione notturna nelle prigioni del Castello. Qui la prosa di Alfonso, che intreccia l'essenzialità dei dati (ma si tratta di dettagli che, di nuovo, se non fossero realistici sarebbero perfettamente congrui all'ambientazione di una novella gotica, come il tema lasciava supporre) all'invadenza emotiva di quel sensibilissimo sismografo che è diventata la sua ansia, tocca uno dei vertici dell'intera narrazione (pp. 40-41):

... ci lasciarono soli chiudendo l'uscio col catenaccio a doppio chiavistello. Dico il vero che quando udii scorrere il paletto negli anelli mi sentii mancare la respirazione e mi sembrò che mettessero il coperchio al mio sepolcro: fui preso da un gelido sudore e, per non cadere, dovetti salire sul parapetto di quella specie di finestra che c'era onde respirare un po' d'aria fresca e pura, ché l'interna mi pareva poca e già corrotta. Quell'unica apertura aveva, come di solito nelle carceri, un tavolato ai lati e in faccia, sporgente all'infuori, il quale, alzandosi per bene, due terzi di detta apertura non permetteva di vedere, a chi stava nell'interno, che una lista di cielo e la cima di un'altra torre quadrata posta in 
mezzo al castello, su cui era un telegrafo. La brezza notturna mi rimise il vigore nelle vene e respirai più largamente. Per buona sorte nel mezzo dell'imposta dell'uscio c'era uno sportellino da cui entrava a rompere le tenebre il barlume di una lampada collocata nell'andito tra noi e la prima cameraccia. Rinchiusi sedici o diciassette che fossimo in un piccolo spazio senza né una panca dove sederci né un poco di paglia ove sdraiarci, la nostra situazione ci pareva molto brutta. A poco a poco, più che il presente, ci mettemmo a considerare l'avvenire. Tutti avevamo di che temerlo: chi per essersi fatto vedere col fucile in mano, chi per essere entrato col popolo nel palazzo di governo, il povero Durini per la sgridata del generale, altri per altre cose ed io forse più di tutti... Alcuni poi erano sdegnati che la città fosse trascorsa a tanto. E mi ricordo che Giberto Borromeo ripeteva sempre che non avrebbe mai perdonato ai Milanesi la follia di voler cacciare l'esercito austriaco con sassi e bastoni.

E sùbito, a chiusura del periodo, la nota di colore che prepara la sequenza contrastiva che seguirà:

Non si poté però a meno di non ridere, quando uno dei nostri, cercando il fazzoletto, si accorse che gli era stato rubato dai Croati che ci scortarono, giacché l'aveva posto in tasca appunto quando dietro di lui si erano messi coloro.

Dopo che «un po' di sonno torbido e interrotto» (p. 44) ha concesso una breve tregua, eccoci al punto. Cambia il turno di guardia, il che consente, con le dovute cautele, di tentare un avvicinamento (p. 45):

Più tardi, cambiato il croato di guardia e sembrandoci che quest'ultimo avesse più cera di galantuomo, che di croato, ci provammo a interrogarlo, regalandogli qualche moneta. Fosse gratitudine o sincerità, ci disse stentatamente però: "Cittadini tenere tutta la città, noi non tenere che bastioni" e non se ne poté tirar altro. L'effetto che ci fecero queste parole è più facile immaginarlo che spiegarlo. Allora tutto quello a cui prima non si dava grande importanza, ci sembrò non solo importante ma favorevolissimo ai nostri desideri. Così le campane ci sembravano suonare più alacremente e annunciar quasi la vittoria dei nostri.

Breve illusione, naturalmente. Ma sufficiente, nella restituzione di un po' d'umanità alla soldataglia austriaca, ad aprire lo spiraglio a qualche speranza. Nella concitata rassegna dei compagni prigionieri il lettore ha incontrato nomi di famiglie illustri, e questo fatto non sarà indif- 
ferente all'occhio di riguardo che si porterà in breve su di loro. A partire dallo stesso Alfonso, naturalmente, per il quale giocherà però decisivamente la richiesta rivolta direttamente a Radetzky (p. 49) dalla giovane moglie (che nella supplica si dichiara incinta: non abbiamo dettagli precisi, ma la prima figlia della coppia, Clementina, nascerà solo l'anno successivo, per cui non è inverosimile pensare che si trattasse di una ben meditata strategia).

La sospirata liberazione porta Alfonso «sullo spianato davanti al castello» (p. 51). Come abbiamo anticipato, ecco lo sguardo diverso che posa sulla colorita marmaglia che presidia la fortezza:

Qui mi si offerse alla vista un gran numero di soldati come accampati, e un po' più in là una catena di sentinelle. Pioveva poco ma l'aria era talmente pregna di umidità che in un momento mi sentii molle fino all'ossa. Quella povera gente chissà da quanto tempo era esposta all'acqua, senza fuochi per non attirar l'attenzione del nemico, in un terreno molle e fradicio: mi sembrava intirizzita, stanchissima e scoraggiata...

Un'ultima sosta la facciamo qui anche noi, per confermarci quel che già abbiamo sondato circa i riferimenti culturali del nostro diarista. Perché al lettore non sarà sfuggita la sintonia con quel singolare manifesto umanitario che, due anni prima delle Cinque giornate, aveva messo in carta a Milano, dov'era ospite in Casa Manzoni, il toscano Giuseppe Giusti. In Sant'Ambrogio, ad accompagnarlo alla funzione pubblica che si conclude con il «lento lento» canto dei soldati settentrionali - «povera gente» qui come nel passo che si è appena stralciato dal diario di Alfonso - era il figlio scapestrato di Manzoni, Filippo, quello appunto che si vedrà deportato a Kufstein. E nel primo brano di questa sequenza abbiamo isolato un altro significativo referto stilistico (pure evidenziato in corsivo), la «faccia arrovellata» dell'ufficiale che mima, terrorizzando i prigionieri, il gesto dell'arma che spara. L'aggettivo, un bapax assoluto nella narrazione di Alfonso, con la sua esibita ricercatezza rinvia senz'altro e direttamente al cap. II del romanzo manzoniano, e cioè alla descrizione di Renzo che, messo sull'avviso da Perpetua, punta con violenta risolutezza su don Abbondio per avere conferma dei suoi sospetti circa l'impossibilità di celebrare il matrimonio: «... corse verso di lui, con un tratto baldanzoso, e con gli occhi arrovellati». Senonché la citazione, per questo arrovellati che non avrà più modo di ripresentarsi nella prosa né nei versi di Manzoni, deriva 
dalla prima edizione del romanzo, la Ventisettana: l'edizione definitiva livellerà infatti gli occhi di Renzo a «stralunati», ${ }^{14}$ secondo la pratica di riduzione del registro espressivo perseguita da Manzoni nella revisione. E tanto ci può bastare per mettere a fuoco la lettura, condotta dunque per tempo dal giovane Litta Modignani, di quella precisa forma del romanzo: la più "milanese", anche, e probabilmente rimasta la prediletta, secondo un gusto che incontrerà anche estimatori ben più illustri (basti, per tacer d'altri, il solo nome di De Sanctis).

Il gioco di contrasti che abbiamo visto esercitato nella "umanizzazione" dei croati trasforma l'intera città, nella chiusura del diario, in un delirio di folla e di entusiasmo. Le sue Cinque giornate sono in realtà tre soltanto, e dell'infausta conclusione della rivolta Alfonso non avrà dunque motivo di parlare. Lo lasciamo quando da San Giovanni sul Muro e da una prima sosta, appena liberato, in palazzo Litta, superando le barricate volge verso palazzo Borromeo, dove incontra il cugino che gli si fa innanzi nella vesta di un banditto da Grand Tour, «armato come un brigante delle Calabrie» (p. 57). E, da lì, verso casa, «là dove i miei pensieri non avean mai lasciato un istante di essere». Fortunato. $\mathrm{E}$ fortunati i lettori che possono aggiungere questa singolare cronaca, così intensamente umana, alle tante pagine già note su un episodio che resta fondamentale nella costruzione della nostra complessa (e fragile) identità storica. 
